\title{
Transient Perturbation Analysis in Digital Radio
}

\author{
Ricardo Jauregui, Marc Pous, Mireya Fernández and Ferran Silva \\ Departament d'Enginyeria Electrònica (DEE), Grup de Compatibilitat Electromagnètica (GCEM), Universitat Politècnica \\ de Catalunya (UPC), Barcelona, Spain.ricardojt@eel.upc.edu \\ ricardojt@eel.upc.edu \\ mpous@eel.upc.edu \\ mireya@eel.upc.edu \\ ferran.silva@upc.edu
}

\begin{abstract}
This paper presents a new simulation methodology to study the effect of radiated transient disturbances on digital communication systems. The procedure is divided in two stages. In the first one, FDTD numerical simulation is used to determine the transient levels coupled to the antennas. In the second stage, these levels are analyzed by means of a signal processing simulation software to determine the degradation caused in the system. The procedure is applied to a DAB system in car when a radiated interference is generated by a transient in a vehicle cable. The results show that the amplitude of the radiated transient coupled signal is a key parameter, but it is also essential to take into account other parameters such as burst duration and frequency.
\end{abstract}

\section{INTRODUCTION}

The need of a more efficient use of the radio electric space next to the growing demand for higher quality levels, led to the birth of digital radios. One of the most important aspects of the radio digitalization is the channel performance improvement. Furthermore, the digital radio using modern transmission, coding and compression techniques allows better sound quality in a smaller bandwidth than the traditional analogue radio.

Nowadays there are four major groups of technologies for digital radio: Digital Audio Broadcasting (DAB), Digital Radio Mundial (DRM), In-Band Channel (IBOC) and Digital Satellite radio (SDARS). Among them, currently the most widespread are the DAB and DRM, which are already in place in many countries in Europe, Asia and America. In the U.S., digital broadcasting is covered mostly with IBOC, and SDARS [1]. In Europe a significant deployment of digital radio is being developed under the Eureka 147 DAB system.

Despite the many advantages and means for error detection and correction, all digital communications systems have a weakness against transient interference which could become a persistent problem for the user. The quality and immunity of digital receivers in front of radiated transient interferences are determined by multiple aspects [2].

At this moment, most of the studies are analyzing the effect of the transient interferences with a single pulse, characterized by parameters like amplitude, rise time and energy. But the errors produced in a real system will not depend only on the single pulse features. Parameters like the burst frequency, duration and interval are as important as the level of the transient signal [2] [3].

In the particular case of a digital radio working in a vehicle, one of the factors that undoubtedly determine the level and the characteristics of the transient disturbance coupled to the digital system are the location of the antennas used by the receiver [3] [4].

In recent years, some studies have been devoted to know the effect of the coupling of transients in different parts of the vehicle through numerical methods [5] [6] . However, none of those studies are able to use numerical methods to analyze completely the effect of radiate transients disturbances in a digital communication system.

In this paper, Finite Difference Time Domain (FDTD) is used to determine the levels coupled to a DAB antenna when a radiated disturbance is produced by a transient in a vehicle cable. Several antennas are considered in different locations of the car frame. Then, these coupled signals are analyzed with a signal processing simulation software to understand the real impact of the interference on the system and to find the most suitable place for the antenna.

\section{Methodology}

The aim of this study is to analyze the effect of a radiated transient burst, generated by a wire inside a car, on a DAB system installed in the vehicle.

In order to evaluate the influence of the antenna location, four different antennas positions are considered. They are placed at different points in the car as follows (Fig 1):
A. Antenna at the front of the vehicle roof (M1).
B. Antenna at the centre of the vehicle roof (M2).
C. Antenna embedded in the rear right side window glass (M3a).
D. Antenna embedded in the rear left side window glass (M3b).




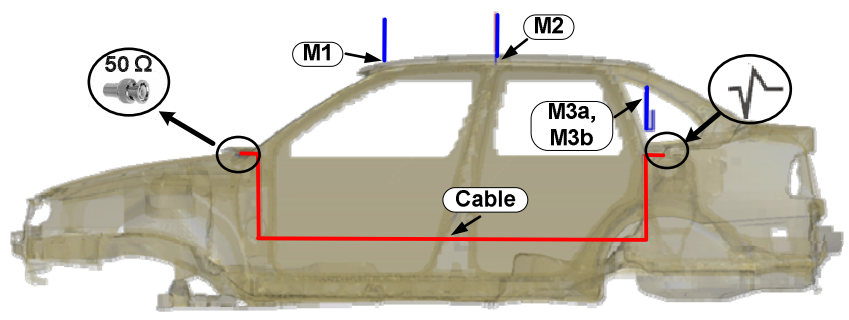

(a)

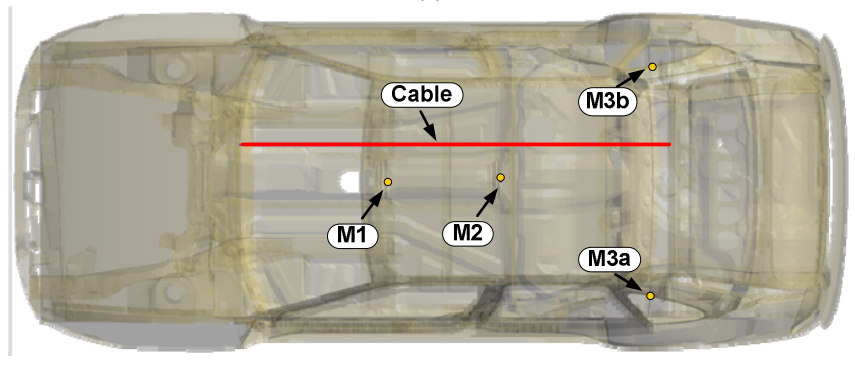

(b)

Fig 1. Different positions of antennas of a digital terrestrial receiver. (a) Side view. (b) Top view.

Fig 1 shows the location of the cable that carries the transient in the vehicle. A typical burst transient defined for EMC testing (EN61000-4-4 [7]) is used. The signal is applied inside the vehicle in a single wire of $2.9 \mathrm{~m}$ in length and $2 \mathrm{~mm}$ wide. To have a more realistic behaviour in the system, the transient signal is generated and captured with a Schölder burst generator. This signal has a bandwidth of approximately $250 \mathrm{MHz}$ and the selected amplitude is $1 \mathrm{kV}$.

A numerical model of the vehicle has been developed to be simulated with the FDTD method. The program used to simulate the vehicle is SEMCAD [8]. The maximum size of mesh used in the car-frame is of $2 \mathrm{~mm}$, the total model has 18 MCells. A Pentium CoreTM 2 Duo 2.66 GB processor and 8Gbyte RAM have been used for the calculation.

The FDTD simulation provides the transient signal coupled in each antenna. Due to simulation time requirements, when the vehicle and the total system are simulated, only one pulse of the burst is considered in the electromagnetic simulation. Before using the FDTD results, it is important to validate the electromagnetic model.

The validation results obtained are presented as a comparison between the simulated and measured waveforms, both in the time domain (Fig 2.). The coupled signal was measured directly with an oscilloscope Tektronix DPO7104 (sampling rate of $2 \mathrm{GSa} / \mathrm{s}$ ). Before being validated the post processing process consists of a low-pass Butterwoth filter with $\mathrm{Fc}=250 \mathrm{MHz}$ applied to both signals (measured \& simulated). The cut-off frequency was selected to match the bandwidth of the transient in the measurements. Additionally a delay of $2.7 \mathrm{~ns}$ was introduced in the simulated waveforms, to compensate for the delays caused by the cables and equipment in the measurements. The magnitude of the delay was estimated by performing a cross-correlation between measured and simulated signals [9].

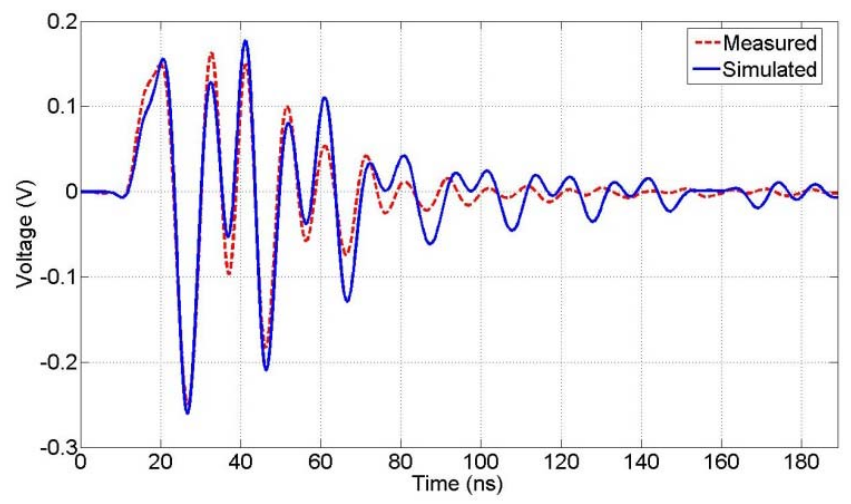

Fig 2. Measured (dashed line) and simulated (solid line) signals for the M1 antenna.

It is clearly observed in both graphs that the simulated and measured signals have a very similar trend. To quantify this similitude the Feature Selective Validation (FSV) method [10] is used in the frequency domain. The GDMt indicator obtained a value of 0.43 (Good). To validate the results in time domain, the Transient Time Domain Validation (TTDV) method [11] is used, obtaining a value 0.101 for the TEA indicator.

Once we have each antenna input, a pre-processing is needed before using a signal processing simulation software to evaluate the effect of the transient in the DAB system. It is very important not to confuse the electromagnetic simulation (FDTD) with the signal processing simulation. The output signal provided by the FDTD numerical simulation is low pass, filtered at the carrier frequency $(100 \mathrm{MHz})$ to eliminate any numerical noise that has occurred in the simulation process. Then, it is demodulated in phase (I) and quadrature (Q). Once we have completed this process, both signals I\&Q have to be filtered again considering the carrier frequency $(100 \mathrm{MHz})$ and the bandwidth of the communication system $(2 \mathrm{MHz})$. After that, the transient is repeated each $8 \mu \mathrm{s}$ along $500 \mu \mathrm{s}$ to produce the burst signal. Finally, a pulse train is generated by repeating the burst each $1 \mathrm{~ms}$, see Fig 3 .

Once this post-processing phase is finished, the resulting I\&Q interference signal is introduced in the signal processing simulation software WinIQSIM [12], to analyze the effect caused by the transient on the system. At this stage, it is necessary to define the normal characteristics of the system, such as numbers of symbols, number of carriers, the reference level used and the white noise level considered in the system. 


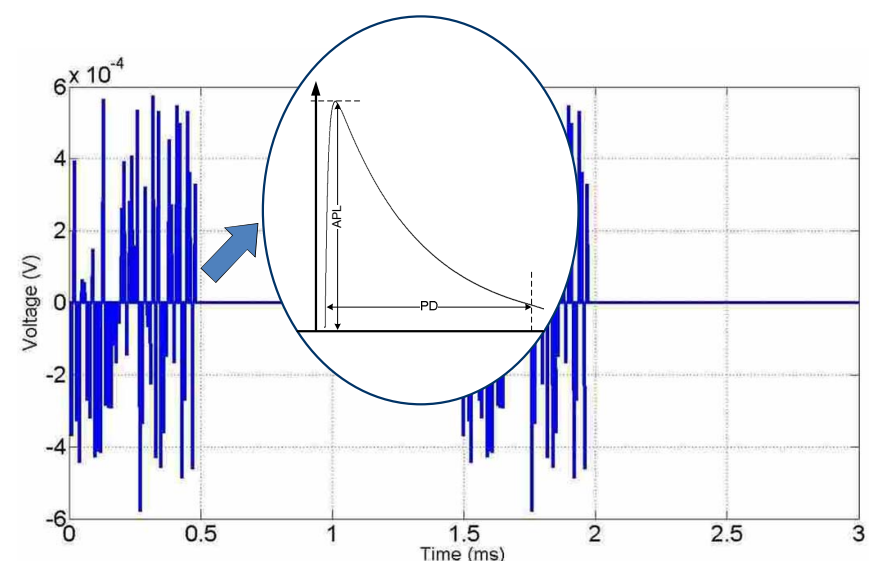

(a)

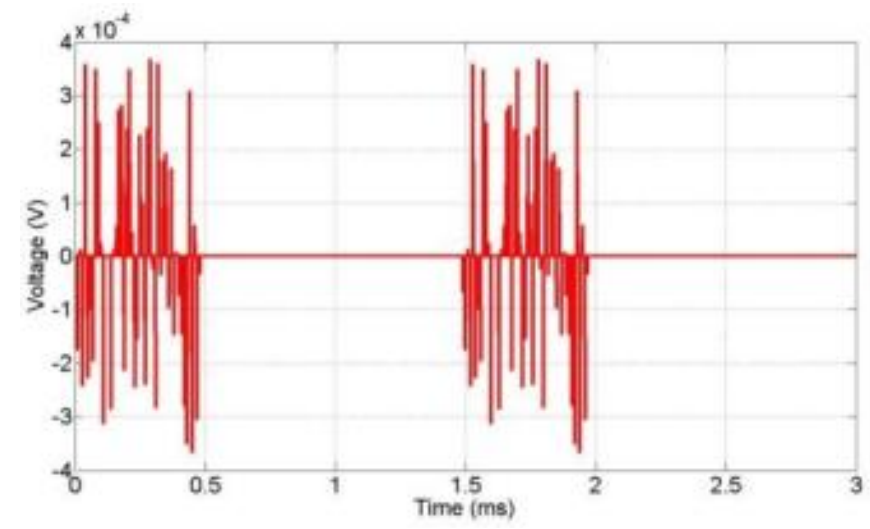

(b)

Fig 3. Transient bursts in (a) phase and (b) quadrature.

For this example, we have defined a generic Orthogonal Frequency Division Multiplexing (OFDM) system with 4QAM modulation, the reason for this simplification are the limitations found in the particular signal processing simulation software used to perform the analysis. This simplification does not jeopardize the application of the proposed method, in a complete QAM scheme, only the overall immunity of the system will be larger. Other details of the system analyzed are: $100 \mathrm{MHz}$ carrier frequency, $2 \mathrm{MHz}$ bandwidth and 20 carriers (instead of the usual 1536 carriers in DAB systems), 5000 symbols, $54 \mathrm{~dB} \mu \mathrm{V}$ reference level and added white noise to obtain $\mathrm{SNR}=30 \mathrm{~dB}$. Fig 4 shows the constellation representation of this system obtained without the transient interference contribution.

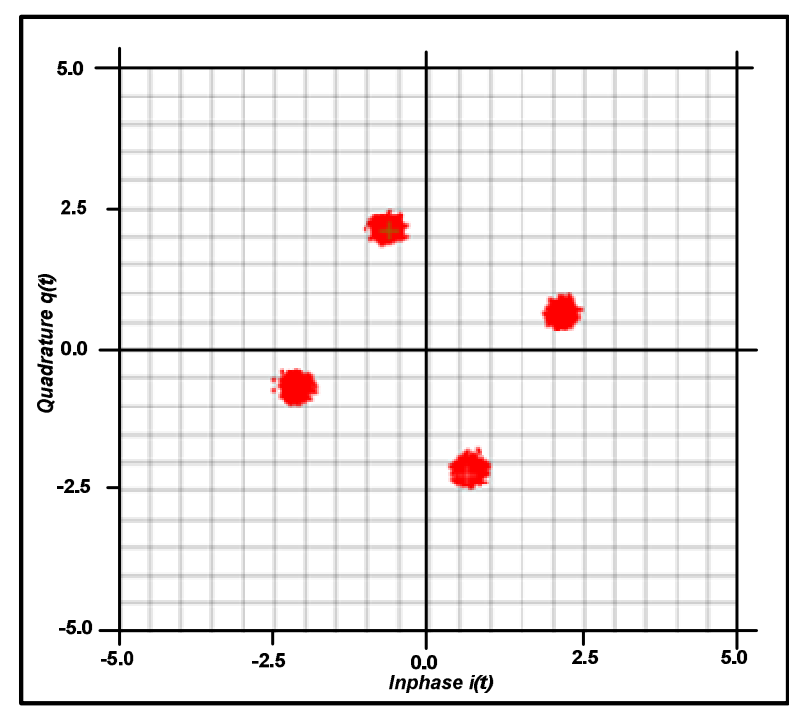

Fig 4. Constellations for OFDM system defined without transient.

\section{RESULTS}

In this section the results obtained for each antenna will be shown. In each antenna location we focus on the one pulse transient signal obtained by the FDTD numerical simulation and the constellation graph obtained by the signal processing simulation software when considering the complete burst.

\section{A. Antenna at the front of the vehicle roof}

The first location selected corresponds to a very common setting in today's cars. Fig 5 shows the results of the FDTD simulation in time domain for the M1 antenna.

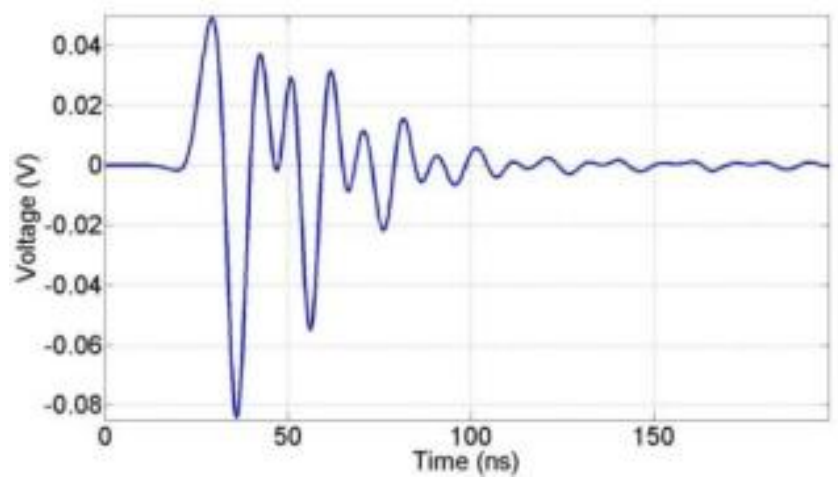

Fig 5. Coupled signal at the antenna located in front of the vehicle roof (M1).

After performing the procedure described in the previous section, Fig 6 shows the constellation of the system related to this antenna location. 


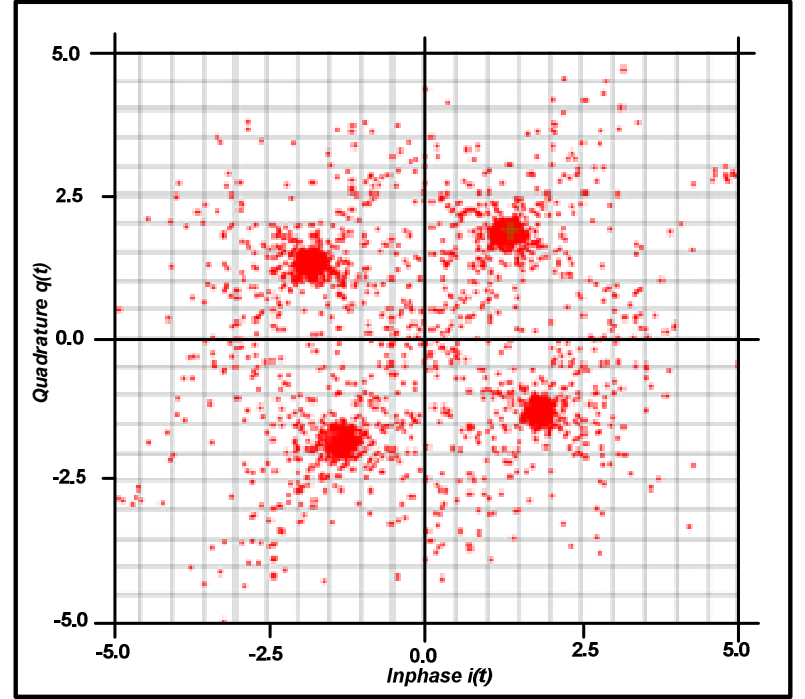

Fig 6. Constellations for OFDM system defined with transient in antenna M1.

Making a visual comparison between the Fig 6 constellation and the one in Fig 4, where there is no transient, we can determine that the interference produces a clear perturbation on the communications system at the M1 antenna location. A significant number of symbols are outside the original areas of decision, concluding that surely there would be major flaws in the system.

\section{B. Antenna at the centre of the vehicle roof}

In the second position, the antenna was located at the center of the car roof (M2, Fig 3). Although this location for the radio antenna is not as popular as several years ago, the case is interesting because the position is still very common for other communication antennas such as GSM or GPS. Fig 7 shows the results of the FDTD simulation.

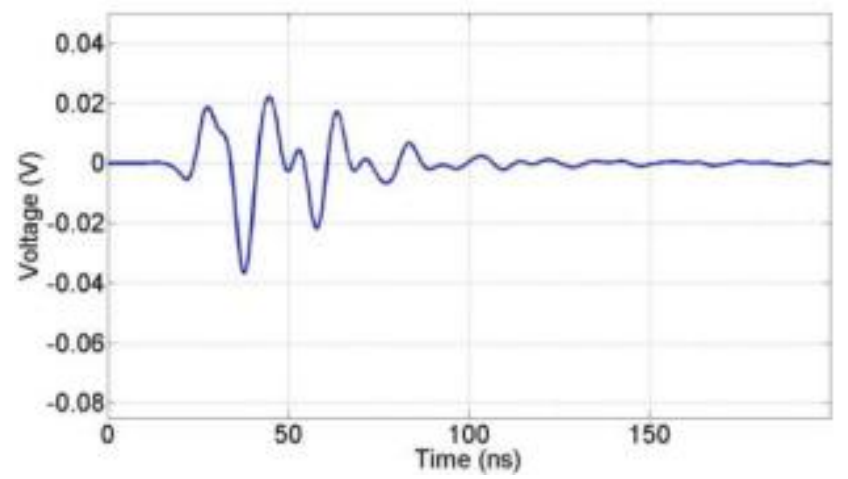

Fig 7. Coupled signal at the antenna located in center of the vehicle roof (M2).

In the previous figure (Fig 7) we observe that the level of the transient signal coupled to the antenna M2 is approximately half of the M1 antenna. The main reason is due to the shielding effect of the roof of the vehicle. Again, the post-processing steps are applied to obtain the constellation diagram that is shown in Fig 8.

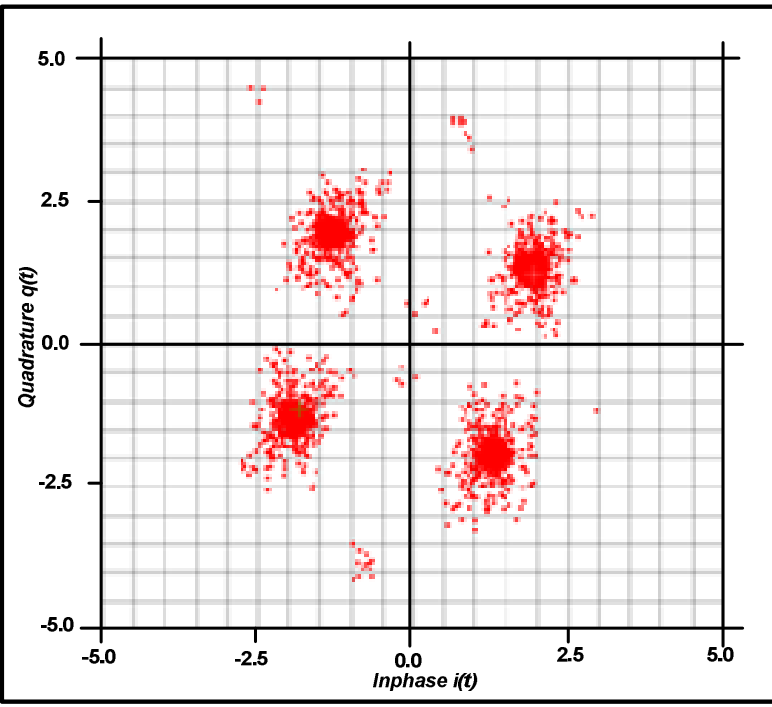

Fig 8. Constellations for OFDM system defined in M2 antenna.

As could be initially expected, compared with case M1, the transient level reduction reduces the nuisance produced in the radio reception. This is shown in Fig 8 with a smaller scattering.

\section{Antennas embedded in the rear side windows glass}

The latter configuration corresponds to the modern technique, that is becoming very popular, in which the antennas are integrated in some vehicle parts. In this case we are considering the antennas printed on the window glass [13] [14]. Specifically, using both lateral rear windows is possible obtain space diversity, that often improve the performance and reception quality.

In this last case two DAB antennas [15] are located in both lateral rear windows: M3a and M3b (Fig 9).

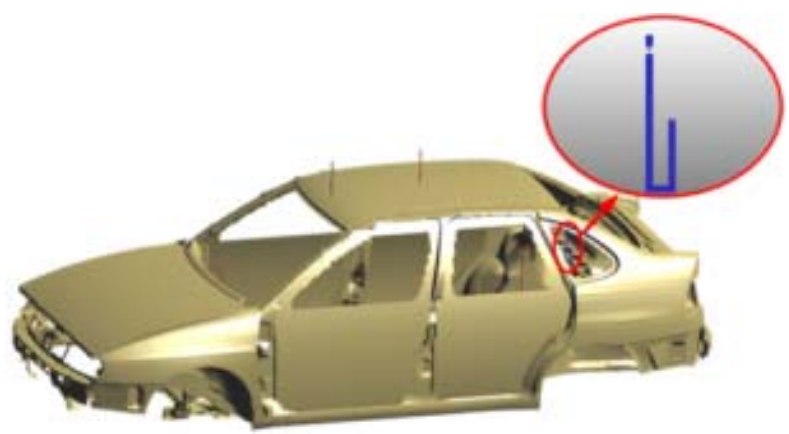

Fig 9. M3a and M3b antenna details in the car model.

The Fig 10 shows the transient signals coupled in both antennas. As expected, the signal coupled to the antenna 
closest to the cable (Fig 10a) is greater than the farther one (Fig 10b) due to radiated signal attenuation inside the car.

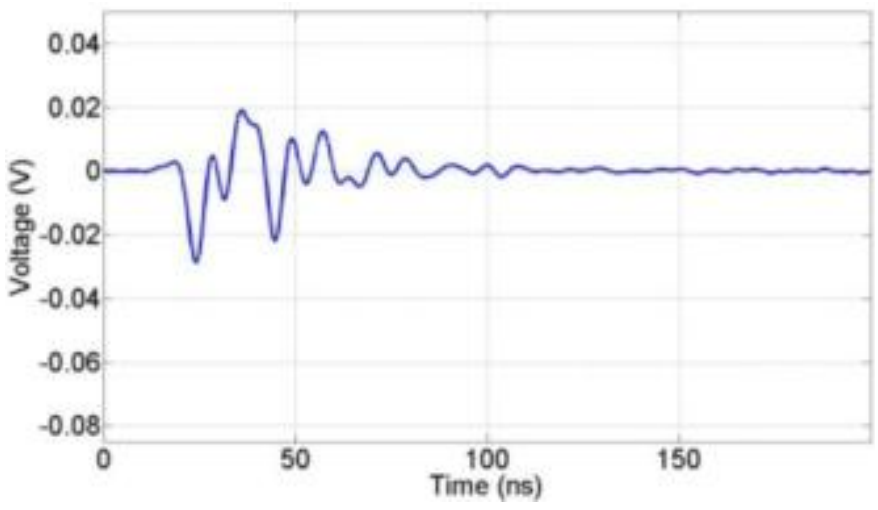

(a)

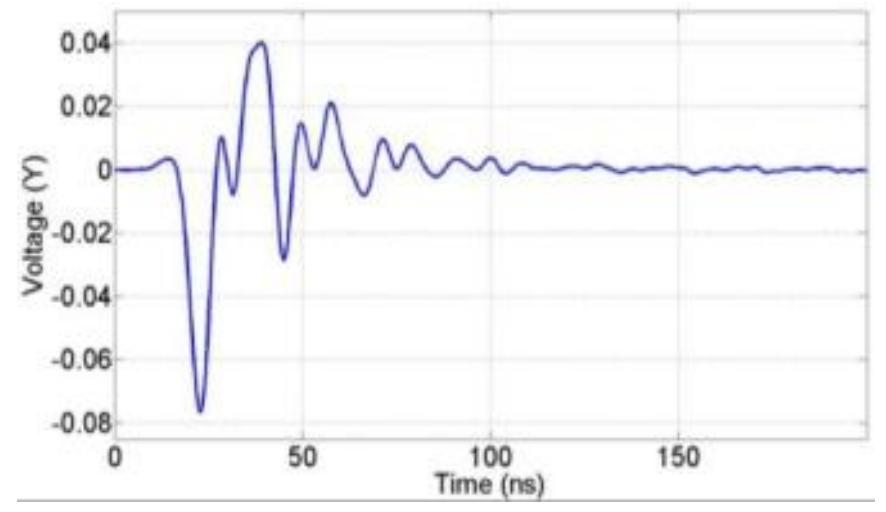

(b)

Fig 10. Coupled signal at the antenna located in the rear right side window glass . (a) M3a antenna. (b) M3b antenna.

The maximum level coupled to the M3b antenna $(\mathrm{Vmax}=0.084 \mathrm{~V})$ is very close to transient received by the M1 antenna $(\mathrm{Vmax}=0.076 \mathrm{~V})$. However, in this case the settling time is smaller, as well as the level of the transient oscillations.

Fig 11 shows the constellation for M3a and M3b antennas once the whole signal process is performed.

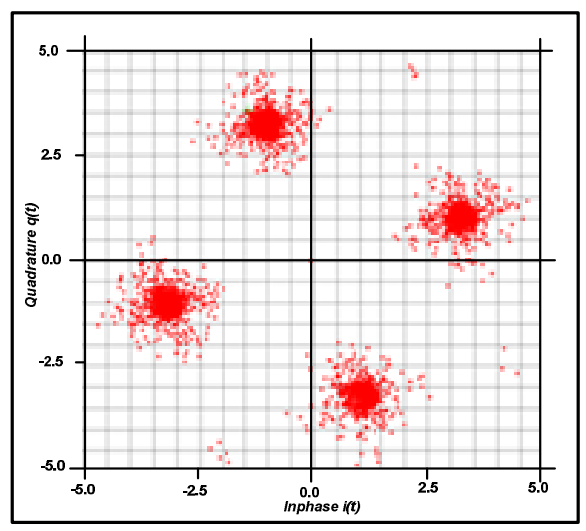

(a)

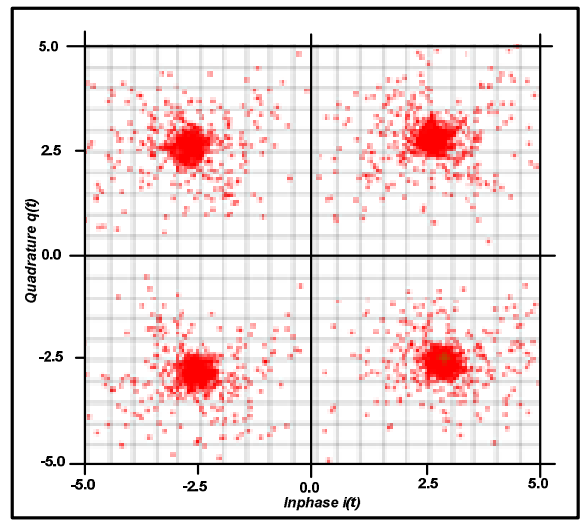

(b)

Fig 11. Constellations for OFDM system defined. (a) M3a antenna. (b) M3b antenna.

As expected, the worst result is obtained in the second case, M3b (Fig 11b), when the antenna is closer to the cable where the disturbance occurs. For the M3a antenna (Fig 11a) the areas are clearly defined and, although there is a small scattering of symbols, a low error rate must be expected.

If the DAB reception quality relies only on M3b antenna, a significant degradation of the communication is likely to be produced due to the transient in the cable. However, as the system is designed to exploit the space diversity, it would be possible to correct the $\mathrm{DAB}$ reception using the M3a antenna, thereby decreasing the amount of errors received.

It is very remarkable that, if we only take into account the peak levels of the coupled transients, the results for M1 and M3b antenna should be similar since the peak levels produced at the antennas by the FDTD simulations are similar. But in this communication system, which causes the greatest perturbation nuisance are not the maximum levels, but the 100 $\mathrm{MHz}$ components, which are very different in both situations. For this reason, although the peak levels are the same, M1 constellation (Fig 6) shows a larger error probability than M3b (Fig 11b). 


\section{CONCLUSIONS}

This paper describes a methodology to analyze the behavior of a communication system against transient interference without the need to do actual measurements in real environments.

The proposed method has two stages, first the radiated transient coupled signal to the communication system is calculated by means of an FDTD numerical simulation. The second stage uses the previous results and, by means of a signal processing simulation software, evaluates the effect the transient interference provokes in the digital communication system.

In this paper, as an example, the $\mathrm{DAB}$ reception degradation by a transient in a vehicle has been analyzed. From the results we can remark that the amplitude of the radiated transient signal is a key parameter, but it is also essential to take into account other parameters such as burst duration and frequency to make a complete analysis of the disturbance.

\section{ACKNOWLEDGMENT}

This work was sponsored in part by the Spanish research project MICINN (DPI2007-63878).

\section{REFERENCES}

[1] N. Koch, "EMC-Influences on Digital Broadcasting Services in Vehicular Environments," Proc. 20th International Zurich Symposium on Electromagnetic Compatibility, 2009, pp. 209-212.

[2] J. Lago-Fernndez and J. Salter, R\&D White Paper Modelling impulsive interference in DVB-T: statistical analysis, test waveforms \& receiver performance.

[3] R. Kronberger, H.K. Lindenmeier, J.F. Hopf, and L.M. Reiter, "Design method for antenna arrays on cars with electrically short elements under incorporation of the radiation properties of the car body," Proc. Digest Antennas and Propagation Society International Symposium IEEE, 1997, pp. 418-421.

[4] L. Low, R. Langley, R. Breden, and P. Callaghan, "Automotive antenna performance and simulation," Proc. First European Conference on Antennas and Propagation EuCAP 2006, 2006, pp. 1-4.

[5] M.P. Sol, "Develop of a method to measure radiated transient signals and analyse its effect over the immunity of digital communication systems," 2009.

[6] P.J. Riu, R. Jauregui, and F. Silva, "Transient Electromagnetic Field Computation in Automotive Environments using FDTD," 2007 IEEE EMC Symposium, USA, 2007.

[7] IEC 61000-4-4:2001, Electromagnetic Compatibility (EMC) Testing and measuraments tecniques - Electric Fast Transient/burst immunity test, 2001.

[8] Schmid \& Partner Engineering AG (SPEAG), SEMCAD. http:\|www.SEMCAD.com, 2000.

[9] R.Jauregui, F.Silva, and P.Riu., "FDTD Analysis of the distribution of a transient field inside a car.," EMC Europe 2007 Paris Francia, 2007.

[10] A.P. Duffy, A.J. Martin, A. Orlandi, G. Antonini, T.M. Benson, and M.S. Woolfson, "Feature selective validation (FSV) for validation of computational electromagnetics (CEM). part I-the FSV method," Electromagnetic Compatibility, IEEE Transactions on, vol. 48, 2006, pp. 449459.

[11] R. Jauregui, P.J. Riu, and F. Silva, "Transient FDTD Simulation Validation," 2010 IEEE EMC Symposium, USA, 2010, 2010.
[12] Rohde\&Schwarz, R\&S WinIQSIM Simulation Software, 2000.

[13] R. Abou-Jaoude and E.K. Walton, "Numerical modeling of on-glass conformal automobile antennas," vol. 46, 1998, pp. 845-852.

[14] L. Low, R. Langley, R. Breden, and P. Callaghan, "Planar Roof Mounted Automotive Antenna," Proc. Loughborough Antennas and Propagation Conference LAPC 2007, 2007, pp. 109-112.

[15] H.K. Lindenmeier, J.F. Hopf, and L.M. Reiter, "Antenna and diversity techniques for broadcast reception in vehicles," Proc. IEEE Antennas and Propagation Society International Symposium AP-S. 1992 Digest. Held in Conjuction with: URSI Radio Science Meeting and Nuclear EMP Meeting, 1992, pp. 1097-1100. 